\title{
WELL POSEDNESS FOR EVOLUTION INCLUSIONS
}

\author{
K. BALACHANDRAN \\ Bharathiar University, Department of Mathematics \\ Coimbatore 641 046, Tamil Nadu, INDIA \\ A. ANGURAJ \\ Gobi Arts College, Department of Mathematics \\ Gobichettipalayam 638453 Tamil Nadu, INDIA
}

(Received March, 1993; revised May, 1994)

\begin{abstract}
We prove the existence of a continuous selection of the multivalued map $\varphi \rightarrow \Phi(\varphi)$ which is the set of all mild solutions of the evolution inclusion

$$
\begin{gathered}
\dot{x}(t) \in A x(t)+F(t, x(t))+\int_{0}^{t} h(t-s) g(x(s)) d s \\
x(0)=\varphi .
\end{gathered}
$$
\end{abstract}

Here $F$ is a multivalued map, Lipschitzian with respect to $x$, and $A$ is the infinitesimal generator of a $C_{0}$-semigroup.

Key words: Well-posedness, Evolution Inclusion, Mild Solution.

AMS (MOS) subject classifications: $\quad 34 \mathrm{~A} 12,34 \mathrm{~A} 60,45 \mathrm{~J} 05,54 \mathrm{C} 65$.

\section{Introduction}

According to a generally accepted terminology in the theory of differential equations, an initial value problem, whose solutions exist, are unique and depend continuously on the initial data, is called well posed. For problems lacking uniqueness, (i.e., those for which any solution through a point, can be embedded in a continuous, single valued family of solutions depending on the initial point) can be considered as the natural extension of the well posedness.

The existence of a continuous map $\varphi \rightarrow x_{\varphi}$, such that $x_{\varphi}$ is a solution of the Cauchy problem

$$
\dot{x} \in F(t, x), x(0)=\varphi
$$

where $F$ is a nonempty set-valued function, Lipschitzian with respect to $x$, was proved first by Cellina in [4]. Then, the same problem for a differential inclusion with Lipschitzian right-hand side defined on an open set, was studied by several authors $[3,4,6,7]$. Well posedness for a differential inclusion on closed sets was proved in [5]. A continuous function $f: X \rightarrow Y$ is said to be a continuous selection of a multivalued map $F: X \rightarrow 2^{Y}$ if $f(x) \in F(X)$ for all $x \in X$. Continuous selections exist due to continuous selection theorems. A detailed study of continuous selection theorems is given in [2]. The existence of a continuous selection of the set-valued 


$$
\dot{x}(t) \in A x(t)+F(t, x(t)), \quad x(0)=\varphi,
$$

function $\varphi \rightarrow \Phi(\varphi)$, where $\Phi(\varphi)$ is the set of all mild solutions of the Cauchy problem is established in $[7,9]$. Here $F$ is Lipschitzian with respect to $x$ and $A$ is the infinitesimal generator of a $C_{0^{-}}$ semigroup.

In this paper, we consider the evolution inclusion of the form

$$
\begin{gathered}
\dot{x}(x) \in A x(t)+F(t, x(t))+\int_{0}^{t} h(t-s) g(x(s)) d s, \\
x(0)=\varphi
\end{gathered}
$$

where $F$ is a set-valued function and $\varphi \in X$. We prove the existence of a continuous selection of the set-valued function $\varphi \rightarrow \Phi(\varphi)$, where $\Phi(\varphi)$ is the set of all mild solutions of the Cauchy problem (1), assuming that $F$ is Lipschitzian with resect to $x$ and $A$ is the infinitesimal generator of a $C_{0}$-semigroup. This work was motivated by the existence of unique mild solution of the evolution integrodifferential equation studied by Ahmed [1].

\section{Preliminaries}

Let $T>0, I=[0, T]$ and denote $\mathcal{L}$ the $\sigma$-algebra of all Lebesgue measurable subsets of $I$. Let $X$ be a real separable Banach space with the norm $\|\cdot\|$. Denote by $\mathscr{B}(X)$ the family of all Borel subsets of $X$. For any subset $A \subset X$ and $x \in X$, we set $d(x, A)=\inf \{\|x-y\|: y \in A\}$. Furthermore, for two closed bounded nonempty subsets $A$ and $B$ of $X$, we denote by $h(A, B)$ the Hausdorff distance from $A$ to $B$, that is, $h(A, B)=\max _{x \in A}\left\{\sup _{x \in A} d(x, B), \sup _{y \in B} d(y, A)\right\}$.

Denote by $C(I, X)$ the Banach space of all continuous functions $x: I \rightarrow X$ endowed with the norm $\|x\|_{\infty}=\sup \{\|x(t)\|: t \in I\}$ and by $\mathcal{L}^{1}(I, X)$, the Banach space of all Bochner integrable functions $x: I \rightarrow X$ with norm $\|x\|_{1}=\int_{0}^{T}\|x(t)\| d t$. A subset $K$ of $\mathscr{L}^{1}(I, X)$ is called decomposable if for every $u$ and $v$ in $K$ and $A \in L$ we have $u \aleph_{A}+v \aleph_{I-A} \in K$, where $\aleph_{A}$ stands for the characteristic function of $A$. Denote by $\mathscr{D}$ the family of all closed nonempty decomposable subsets of $\ell^{1}(I, X)$.

Let $X$ be a separable Banach space and $\{G(t): t \geq 0\} \subset \mathcal{L}(X, X)$ be a strongly continuous semigroup of bounded linear operators from $X$ to $X$ having infinitesimal generator $A$. Consider the Cauchy problem (1) where $\varphi \in X$ and $F: I \times X \rightarrow 2^{X}$ is a set-valued function satisfying the following hypotheses:

(i) $\quad F$ is the $\mathcal{L} \otimes \mathscr{B}(X)$ measurable,

(ii) there exists $k \in \mathcal{L}^{1}(I, R)$ such that $h(F(t, x), F(t, y)) \leq k(t)\|x-y\|$, for all $x, y \in X$ a.e. in $I$,

(iii) there exists $\mu \in \mathcal{L}^{1}(I, R)$ such that $d(0, F(t, 0)) \leq \mu(t), t \in I$ a.e.,

(iv) $g: X \rightarrow X$ be a continuous function and there exists a constant $C>0$ such that $\|g(x)\| \leq C(1+\|x\|)$ and $\|g(x)-g(y)\| \leq C\|x-y\|$, for all $x, y \in X$,

(v) $\quad h \in \mathcal{L}^{1}(I, R)$ and there exists a constant $H \in \mathcal{L}^{1}(I, R)$ such that for each pair $s, t \in I$ with $s<t, \int_{s}^{t}|h(\tau)| d \tau \leq \int_{0}^{T}|h(\tau)| d \tau=H$.

Definition 1: A function $x(\cdot, \varphi): I \rightarrow X$ is called a mild solution of the Cauchy problem (1) if there exists $f(\cdot, \varphi) \in \mathcal{L}^{1}(I, X)$ such that

(i) $\quad f(t, \varphi) \in F(t, x(t, \varphi))$ for almost all $t \in I$; and 


$$
\left.x(t, \varphi)=G(t) \varphi+\int_{0}^{t} G(t-\tau) f(\tau, \varphi) d \tau+\int_{0}^{t} G(t-\tau)\left(\int_{0}^{\tau} h(\tau-s) g(x(s, \varphi)) d s\right) d \tau\right)
$$

for each $t \in I$.

We denote by $\Phi(\varphi)$ the set of all mild solutions of (1).

Let $S$ be a separable metric space. A set-valued function $G: S \rightarrow 2^{X}$ is called lower semicontinuous if the set $\{s \in S: G(s) \subset C\}$ is closed in $S$ for any closed $C \subset X$. The following two lemmas are used in the sequel.

Lemma 1:[7] Let $F: I \times S \rightarrow 2^{X}$ be $L \otimes \mathscr{B}(S)$ measurable and lower semicontinuous in $s$. Then, the function $s \rightarrow G_{F}(s)$ given by

$$
G_{F}(s)=\left\{v \in \mathcal{L}^{1}(I, X): v(t) \in F(t, s) \text { a.e. in } I\right\}
$$

is lower semicontinuous from $S$ into $\mathscr{D}$ if and only if there exists a continuous function $\mu: S \rightarrow \mathcal{L}^{1}(I, R)$ such that for every $s \in S, \mu(s)(t) \leq d(0, F(t, s))$ a.e. in $I$.

Lemma 2: [7] Consider a lower semicontinuous function $G: S \rightarrow \mathscr{D}$ and assume that $p: S \rightarrow \mathcal{L}^{1}(I, X)$ and $q: S \rightarrow \mathcal{L}^{1}(I, R)$ are continuous functions and for every $s \in S$ the set

$$
H(s)=c l\{u \in G(s):\|u(t)-p(s)(t)\| \leq q(s)(t) \text { a.e. in } I\}
$$

is nonempty. Then the function $H: S \rightarrow \mathscr{D}$ is lower semicontinuous, so it has a continuous selection.

\section{Well Posedness}

Theorem 1: Let $A$ be the infinitesimal generator of a $C_{0}$-semigroup $\{G(t): t \geq 0\}$ and let the hypotheses $(i)-(v)$ be satisfied. Then, there exists a function $x(\cdot, \cdot): I \times X \rightarrow X$ such that

(i) $\quad x(\cdot, \varphi) \in \Phi(\varphi)$ for every $X$; and

(ii) $\varphi \rightarrow x(\cdot, \varphi)$ is continuous from $X$ into $C(I, X)$.

Proof: Let $M=\sup \{\|G(t)\|: t \in I\}$, and for $\varphi \in X$ defined $x_{0}(\cdot, \varphi): I \rightarrow X$ by $x_{0}(t, \varphi)=$ $G(t) \varphi$. Clearly $\varphi \rightarrow x_{0}(\cdot, \varphi)$ is continuous from $X$ to $C(I, X)$. For each $\varphi \in X$, let $\alpha(\varphi): I \rightarrow R$ be given by

$$
\alpha(\varphi)(t)=\mu(t)+k(t)\left\|x_{0}(t, \varphi)\right\|
$$

Clearly, $\alpha(\cdot)$ is continuous from $X$ to $\mathcal{L}^{1}(I, R)$. Moreover, for each $\varphi \in X$,

$$
d\left(0, F\left(t, x_{0}(t, \varphi)\right) \leq \beta(t)+k(t)\left\|x_{0}(t, \varphi)\right\|=\alpha(\varphi)(t) .\right.
$$

Let $\epsilon>0$ be fixed, and for $n \in N$, set $\epsilon_{n}=\epsilon / 2^{n+1}$. Now define

$$
\begin{gathered}
G_{0}: X \rightarrow 2^{\mathcal{L}(I, X)} \text { and } H_{0}: X \rightarrow 2^{\mathcal{L}(I, X)} \text { by } \\
G_{0}(\varphi)=\left\{v \in \mathcal{L}^{1}(I, X): v(t) \in F\left(t, x_{0}(t, \varphi)\right) \text { a.e. } t \in I\right\} \text { and } \\
H_{0}(\varphi)=c l\left\{v \in G_{0}(\varphi):\|v(t)\| \leq \alpha(\varphi)(t)+\epsilon_{0} \text { a.e. } t \in I\right\}
\end{gathered}
$$

Clearly by Lemma $1, G_{0}(\cdot)$ is lower semicontinuous from $X$ into $\mathscr{D}$ and $H_{0}(\varphi) \neq \emptyset$ for each $\varphi \in X$, and, hence by Lemma 2 , there exists a continuous function $h_{0}: X \rightarrow \mathcal{L}^{1}(I, X)$, which is a 
continuous selection of $H_{0}(\cdot)$.

For each $\varphi \in X$, let $\beta(\varphi): I \rightarrow R$ be given by $\beta(\varphi)=H C\left(1+\left\|x_{0}(t, \varphi)\right\|\right)$. Clearly, $\beta(\cdot)$ is continuous from $X$ to $\mathcal{L}^{1}(I, R)$. Let $K(t)=k(t)+C H$ and set $m(t)=\int_{0}^{t} K(\tau) d \tau$. For $n \geq 1$, define $\Gamma_{n}: X \rightarrow \mathcal{L}^{1}(I, R)$ by

$$
\begin{gathered}
\Gamma_{n}(\varphi)(t)=M^{n} \int_{0}^{t} \alpha(\varphi(s)) \frac{[m(t)-m(s)]^{n-1}}{(n-1) !} d s \\
+M^{n} \int_{0}^{t} \beta(\varphi)(s) \frac{[m(t)-m(s)]^{n-1}}{(n-1) !} d s \\
+M^{n} t\left(\sum_{i=0}^{n} \epsilon_{i}\right) \frac{[m(t)]^{n-1}}{(n-1) !}, \quad t \in I .
\end{gathered}
$$

Set $f_{0}(t, \varphi)=h_{0}(\varphi)(t)$ and $g_{0}(x(t, \varphi))=g\left(x_{0}(t, \varphi)\right)$. Since $x_{0}(\cdot, \varphi)$ is continuous, $g_{0}(x(\cdot, \varphi))$ is also continuous. Define

$$
x_{1}(t, \varphi)=G(t) \varphi+\int_{0}^{t} G(t-\tau) f_{0}(\tau, \varphi) d \tau+\int_{0}^{t} G(t-\tau)\left(\int_{0}^{\tau} h(\tau-s) g_{0}(x(s, \varphi)) d s\right) d \tau, \quad t \in I
$$

Then, $f_{0}(t, \varphi) \in F\left(t, x_{0}(t, \varphi)\right), \quad\left\|f_{0}(t, s)\right\| \leq \alpha(s)(t)+\epsilon_{0}, \quad$ and $\quad\left\|g_{0}(x(t, \varphi))\right\|=\| g\left(x_{0}(t, \varphi) \|\right.$ $\leq C\left(1+\left\|x_{0}(t, \varphi)\right\|\right)$. For $t \in I \backslash\{0\}$ and by Fubini's theorem,

$$
\begin{aligned}
& \left\|x_{1}(t, \varphi)-x_{0}(T, \varphi)\right\| \leq \int_{0}^{t}\|G(t-\tau)\|\left\|f_{0}(\tau, \varphi)\right\| d \tau \\
& +\int_{0}^{t}\left(\int_{0}^{t}\|G(t-\tau)\||h(\tau-s)| d \tau\right) \| g_{0}(x(s, \varphi) \| d s \\
& \leq M \int_{0}^{t}\left\|f_{0}(\tau, \varphi)\right\| d \tau+M \int_{0}^{t} H\left\|g\left(x_{0}(\tau, \varphi)\right)\right\| d \tau \\
& \leq M \int_{0}^{t}\left(\alpha(\varphi)(\tau)+\epsilon_{0}\right) d \tau+M \int_{0}^{t} H C\left(1+\left\|x_{0}(\tau, \varphi)\right\|\right) d \tau \\
& \leq M \int_{0}^{t} \alpha(\varphi)(\tau) d \tau+M T \epsilon_{0}+M \int_{0}^{t} \beta(\varphi)(\tau) d \tau<\Gamma_{1}(\varphi)(t) .
\end{aligned}
$$

We claim that there exist three sequence $\left\{f_{n}(\cdot, \varphi)\right\}_{n \in N},\left\{x_{n}(\cdot, \varphi)\right\}_{n \in N}$ and $\left\{g_{n}(x(\cdot, \varphi))\right\}_{n \in N}$ such that for each $n \geq 1$ the following conditions are satisfied.

(a) $\varphi \rightarrow \bar{f}_{n}(\cdot, \varphi)$ is continuous from $X$ into $\mathcal{L}^{1}(I, X)$,

(b) $\quad f_{n}(t, \varphi) \in F\left(t, x_{n}(t, \varphi)\right)$ for each $\varphi \in X$ and a.e. $t \in I$,

(c) $\quad\left\|f_{n}(t, \varphi)-f_{n-1}(t, \varphi)\right\| \leq K(t) \Gamma_{n}(\varphi)(t)$,

(d) $\quad g_{i}(x(\cdot, \varphi))=g\left(x_{i}(\cdot, \varphi)\right)$ and $g_{n}(x(\cdot, \varphi))$ is continuous from $X$ into $C(X, X)$ for $i=1,2, \ldots$,

(e) $\quad\left\|g_{n}(x(t, \varphi))-g_{n-1}(x(t, \varphi))\right\| \leq K(t) \Gamma_{n}(\varphi)(t)$, 


$$
\begin{aligned}
& x_{n+1}(t, \varphi)=G(t) \varphi+\int_{0}^{t} G(t-\tau) \delta f_{n}(\tau, \varphi) d \tau+\int_{0}^{t} G(t-\tau)\left(\int_{0}^{\tau} h(\tau-s)\right. \\
& \left.g_{n}(x(s, \varphi)) d s\right) d \tau
\end{aligned}
$$

Suppose that we already constructed $f_{1}, f_{2}, \ldots, f_{n}, x_{1}, x_{2}, \ldots, x_{n}$ and $g_{1}, g_{2}, \ldots, g_{n}$ satisfying $(a)-(e)$. Define $x_{n+1}(\cdot, \varphi): I \rightarrow X$ by

$$
x_{n+1}(t, \varphi)=G(t) \varphi+\int_{0}^{t} G(t-\tau) f_{n}(\tau, \varphi) d \tau+\int_{0}^{t} G(t-\tau)\left(\int_{0}^{\tau} h(\tau-s) g_{n}(x(s, \varphi)) d s\right) d \tau .
$$

Then, for $t \in I \backslash\{0\}$, we have that

$$
\begin{gathered}
\left\|x_{n+1}(t, \varphi)-x_{n}(t, \varphi)\right\| \leq \int_{0}^{t}\|G(t-u)\|\left\|f_{n}(u, \varphi)-f_{n-1}(u, \varphi)\right\| d u \\
+\int_{0}^{t}\|G(t-u)\|\left(\int_{0}^{u}|h(u-s)|\left\|g_{n}(x(s, \varphi))-g_{n-1}(x(s, \varphi))\right\| d s\right) d u \\
\leq M \int_{0}^{t} k(u)\left\|x_{n}(u, \varphi)-x_{n-1}(u, \varphi)\right\| d u \\
+M \int_{0}^{t} C H\left\|x_{n}(u, \varphi)-x_{n-1}(u, \varphi)\right\| d u
\end{gathered}
$$

(by Fubini's theorem and making use of $(i v)$ and $(v)$ )

$$
\begin{gathered}
\leq M \int_{0}^{t}(k(u)+C H)\left\|x_{n}(u, \varphi)-x_{n-1}(u, \varphi)\right\| d u \\
\leq M \int_{0}^{t} K(u) \Gamma_{n}(\varphi)(u) d u
\end{gathered}
$$

By making use of calculations provided in [2] we get

$$
\begin{aligned}
\left\|x_{n+1}(t, \varphi)-x_{n}(t, \varphi)\right\| \leq M^{n+1} \int_{0}^{t} \alpha(s)(\tau) \frac{[m(t)-m(\tau)]^{n}}{n !} d \tau \\
+M^{n+1} T\left(\sum_{i=0}^{n} \epsilon_{i}\right) \frac{[m(t)]^{n}}{n !}+M^{n+1} \int_{0}^{t} \beta(s)(\tau) \frac{[m(t)-m(\tau)]^{n}}{n !} d \tau \\
\leq \Gamma_{n+1}(\varphi)(t)
\end{aligned}
$$

and $d\left(f_{n}(t, \varphi), F\left(t, x_{n+1}(t, \varphi)\right) \leq k(t)\left\|x_{n+1}(t, \varphi)-x_{n}(t, \varphi)\right\|\right.$

$$
\begin{gathered}
\leq K(t)\left\|x_{n+1}(t, \varphi)-x_{n}(t, \varphi)\right\| \\
\leq K(t) \Gamma_{n+1}(\varphi)(t) .
\end{gathered}
$$


Define $G_{n+1}: X \rightarrow 2^{\mathcal{L}(I, X)}$ and $H_{n+1}: X \rightarrow 2^{\mathcal{L}(I, X)}$ by

$$
\begin{gathered}
G_{n+1}=\left\{v \in \mathcal{L}^{1}(I, X): v(t) \in F\left(t, x_{n+1}(t, \varphi)\right) \text { a.e. in } I\right\} \text { and } \\
H_{n+1}=c l\left\{v \in G_{n+1}(\varphi):\left\|v(t)-f_{n}(t, \varphi)\right\| \leq K(t) \Gamma_{n+1}(\varphi)(t) \text { a.e. in } I\right\} .
\end{gathered}
$$

Again, by Lemma $1, G_{n+1}(\cdot)$ is lower semicontinuous from $S$ into $\mathfrak{D}$ and $H_{n+1}(\varphi)$ is not empty for each $\varphi \in X$. Hence, by Lemma 2 there exists $h_{n+1}: X \rightarrow \ell^{1}(I, X)$ as a continuous selection of $H_{n+1}(\cdot)$. Then, $f_{n+1}(t, \varphi)=h_{n+1}(\varphi)(t)$ satisfies the conditions $(a)-(c)$. Also

$$
\begin{gathered}
\left\|g_{n+1}(x(t, \varphi))-g_{n}(x(t, \varphi))\right\|=\| g\left(x_{n+1}(t, \varphi)\right)-g\left(x_{n}(t, \varphi) \|\right. \\
\leq C\left\|x_{n+1}(t, \varphi)-x_{n}(t, \varphi)\right\| \\
\leq K(t)\left\|x_{n+1}(t, \varphi)-x_{n}(t, \varphi)\right\| \\
\leq K(t) \Gamma_{n+1}(\varphi)(t) .
\end{gathered}
$$

Therefore, $g_{n+1}$ satisfies $(e)$ and $(f)$ and since $x_{n}(\cdot, \varphi)$ is continuous, $g_{n+1}(t, \varphi)$ is continuous.

Now,

$$
\begin{gathered}
\left\|f_{n}(\cdot, \varphi)-f_{n-1}(\cdot, \varphi)\right\|_{1}=\int_{0}^{T}\left\|f_{n}(u, \varphi)-f_{n-1}(u, \varphi)\right\| d u \\
\leq \int_{0}^{T} k(u)\left\|x_{n}(u, \varphi)-x_{n-1}(u, \varphi)\right\| d u \\
\leq \int_{0}^{T} K(u) \Gamma_{n}(\varphi)(u) d u \\
\leq M^{n} \int_{0}^{\tau} \alpha(\varphi)(u) \frac{[m(T)-m(u)]^{n}}{n !} d u \\
\quad+M^{n} T\left(\sum_{i=0}^{n} \epsilon_{i} \frac{[m(t)]^{n}}{n !}\right. \\
+M^{n} \int_{0}^{T} \beta(\varphi)(u) \frac{[m(T)-m(u)]^{n}}{n !} d u \\
\leq \frac{\left[M\left\|K_{1}\right\|\right]^{n}}{n !}\left[\|\alpha(\varphi)\|_{1}+\|\beta(\varphi)\|_{1}+T \epsilon\right]
\end{gathered}
$$

Since $\varphi \rightarrow\|\alpha(\varphi)\|_{1}$ and $\varphi \rightarrow\|\beta(\varphi)\|_{1}$ are continuous it is locally bounded. Therefore, $\left\{f_{n}(\cdot, \varphi)\right\}_{n \in N}$ is a Cauchy sequence in $\mathcal{L}^{1}(I, X)$. If $f(\cdot, \varphi) \in \mathcal{L}^{1}(I, X)$ is the limit of $\left\{f_{n}(\cdot, \varphi)\right\}_{n \in N}$, then $\varphi \rightarrow f(\cdot, \varphi)$ is continuous from $X$ into $\mathcal{L}^{1}(I, X)$.

Similarly,

$$
\begin{gathered}
\left\|g_{n}(x(\cdot, \varphi))-g_{n-1}(x(\cdot, \varphi))\right\|_{1} \leq \int_{0}^{T} C\left\|x_{n}(u, \varphi)-x_{n-1}(u, \varphi)\right\| d u \\
\leq \int_{0}^{T} K(u) \Gamma_{n}(\varphi)(u) d u
\end{gathered}
$$




$$
\leq \frac{\left[M\left\|K_{1}\right\|\right]^{n}}{n !}\left(\|\alpha(\varphi)\|_{1}+\|\beta(\varphi)\|_{1}+T \epsilon\right)
$$

and so, as previously, $\left\{g_{n}(x(\cdot, \varphi))\right\}_{n \in N}$ is a Cauchy sequence in $C(X, X)$. If $g(x(\cdot, \varphi)) \in$ $C(X, X)$ is its limit, then $x(\cdot, \varphi) \rightarrow g(x(\cdot, \varphi))$ is continuous from $X$ into $C(X, X)$.

On the other hand,

$$
\begin{gathered}
\left\|x_{n+1}(\cdot, \varphi)-x_{n}(\cdot, \varphi)\right\|_{\infty} \leq \int_{0}^{T} M\left\|f_{n}(u, \varphi)-f_{n-1}(u, \varphi)\right\| d u \\
\quad+M H \int_{0}^{T}\left\|g_{n}(x(u, \varphi))-g_{n-1}(x(u, \varphi))\right\| d u \\
\leq M\left\|f_{n}(u, \varphi)-f_{n-1}(u, \varphi)\right\|_{1}+M H\left\|g_{n}(x(u, \varphi))-g_{n-1}(x(u, \varphi))\right\|_{1} \\
\leq M \int_{0}^{T}[K(u)+C H]\left\|x_{n}(u, \varphi)-x_{n-1}(u, \varphi)\right\| d u \\
\leq \frac{\left[M\left\|K_{1}\right\|\right]^{n}}{n !}\left[M\|\alpha(\varphi)\|_{1}+M\|\beta(\varphi)\|_{1}+M T \epsilon\right] .
\end{gathered}
$$

Hence, $\left\{x_{n}(\cdot, \varphi)\right\}_{n \in N}$ is a Cauchy sequence in $C(I, X)$. If $x(\cdot, \varphi) \in C(I, X)$ is its limit then it follows that $\varphi \rightarrow x(\cdot, \varphi)$ is continuous from $X$ into $C(I, X)$.

Since $x_{n}(\cdot, \varphi)$ converges to $x(\cdot, \varphi)$ uniformly, and $d\left(F_{n}(t, \varphi), F(t, x(t, \varphi))\right) \leq$ $K(t)\left\|x_{n}(t, \varphi)-x(t, \varphi)\right\|$, the limit of a subsequence $\left\{f_{n_{k}}\right\}_{k \in N}$ of $\left\{f_{n}\right\}_{n \in N}$ converges pointwise to $f$, so we obtain $f(t, \varphi) \in F(t, x(t, \varphi))$ for $\varphi \in X$ and $t \in I$ a.e.

Furthermore, $g_{n}(x(\cdot, \varphi))$ converges to $g(x(\cdot, \varphi))$ uniformly, passing the limit in the condition (f) we obtain

$$
\begin{gathered}
x(t, \varphi)=G(t) \varphi+\int_{0}^{t} G(t-\tau) f(\tau, \varphi) d \tau \\
+\int_{0}^{t} G(t-\tau)\left(\int_{0}^{\tau} h(\tau-s) g(x(s, \varphi)) d s\right) d \tau \text { for each } t \in I .
\end{gathered}
$$

Therefore, $x(\cdot, \varphi) \in \Phi(\varphi)$ for every $\varphi \in X$ and the proof is complete.

\section{Acknowledgement}

The authors are thankful to Professor Jewgeni H. Dshalalow for the improvement of the paper.

\section{References}

[1] Ahmed, N.U., Nonlinear evolution equations on Banach space, J. Appl. Math. Stoch. Anal. 4 (1991), 187-202.

[2] Aubin, J.P. and Cellina, A., Differential Inclusions, Springer-Verlag, Berlin 1987.

[3] Bressen, A., Cellina, A. and Fryskowski, A., A class of absolute retracts in spaces of 
integrable functions, Proc. Amer. Math. Soc. 384 (1991), 413-418.

[4] Cellina, A., On the set of solutions to Lipschitzian differential inclusions, Diff. Integ. Eqns. 1 (1988), 495-500.

[5] Cellina, A. and Staicu, V., Well posedness for differential inclusions on closed sets, J. Diff. Eqns. 92 (1991), 2-13.

[6] Cellina, A. and Ornelas, A., Representation of the attainable set for Lipschitzian differ ential inclusions, Rocky Mountain J. Math. 22 (1992), 117-124.

[7] Colombo, R.M., Fryskowski, A., Rzezuchowski, T. and Staicu, V., Continuous selections of solutions sets of Lipschitzian differential inclusions, Funkcial. Ekvac. 34 (1991), 321-330.

[8] Staicu, V., Continuous selections of solution sets to evolution equations, Proc. Amer. Math. Soc. 113 (1991), 403-413.

[9] Staicu, V., Well Posedness for Differential Inclusions, Ph.D. Thesis, SISSA, Trieste 1990. 


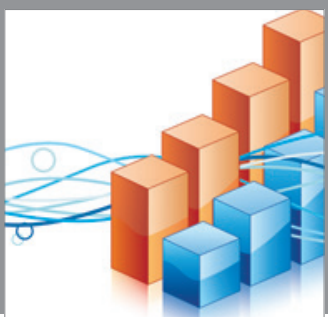

Advances in

Operations Research

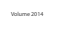

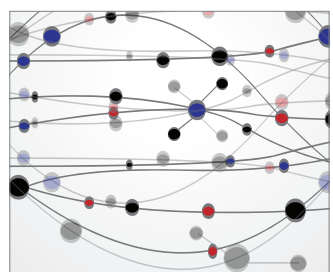

\section{The Scientific} World Journal
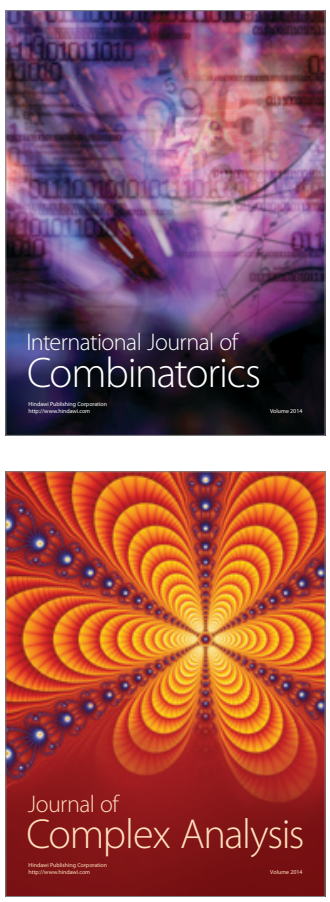

International Journal of

Mathematics and

Mathematical

Sciences
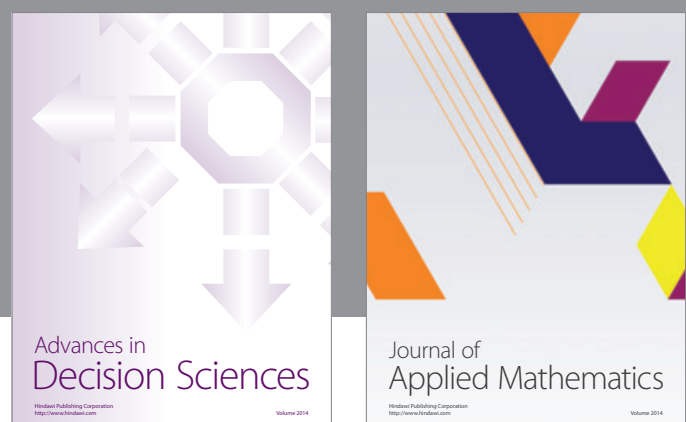

Journal of

Applied Mathematics
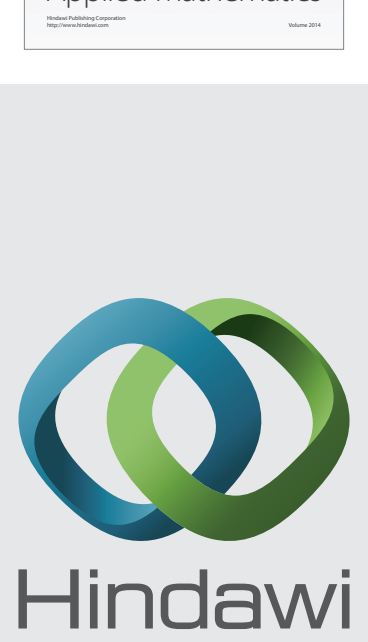

Submit your manuscripts at http://www.hindawi.com
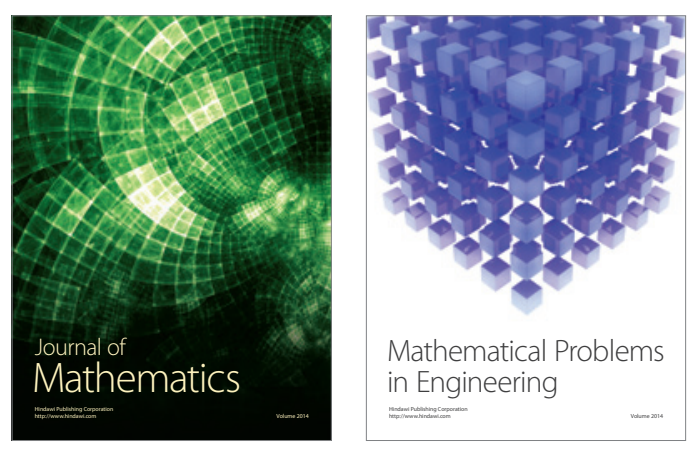

Mathematical Problems in Engineering
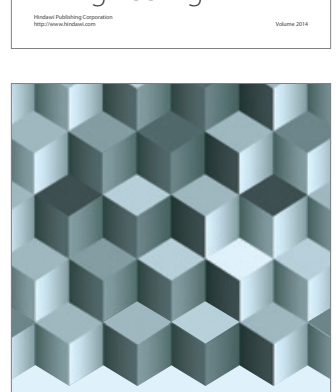

Journal of

Function Spaces
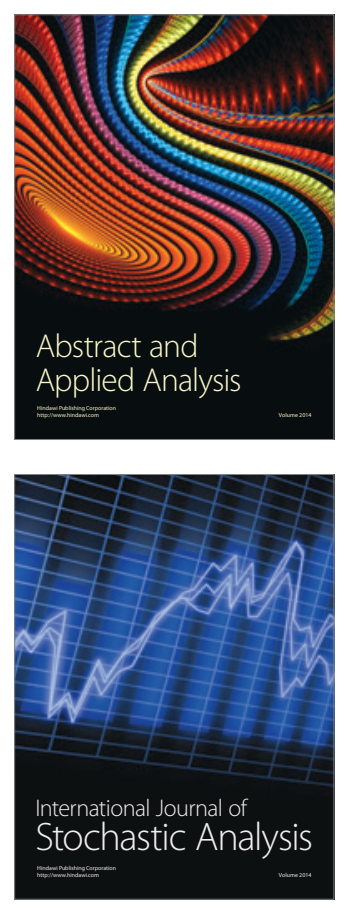

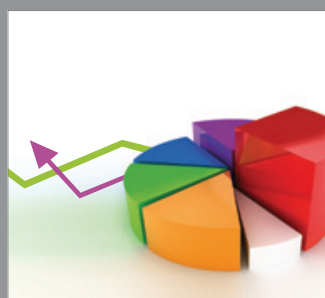

ournal of

Probability and Statistics

Promensencen
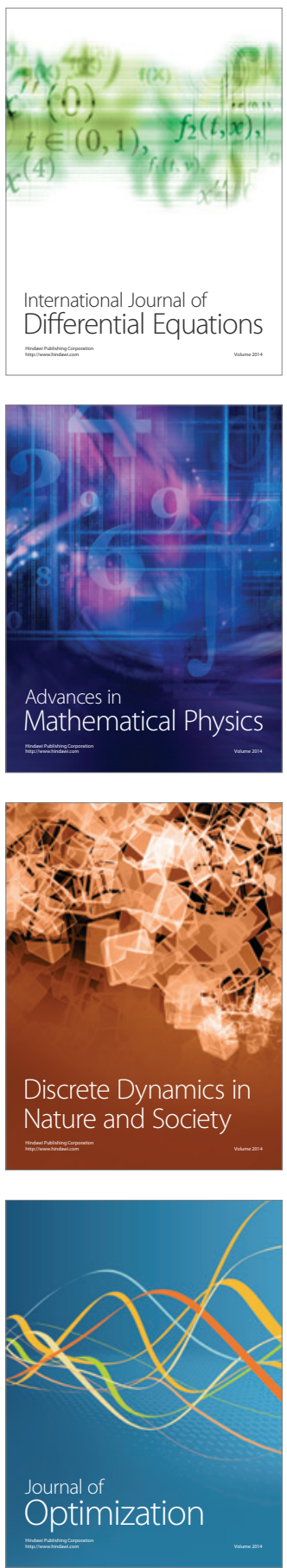